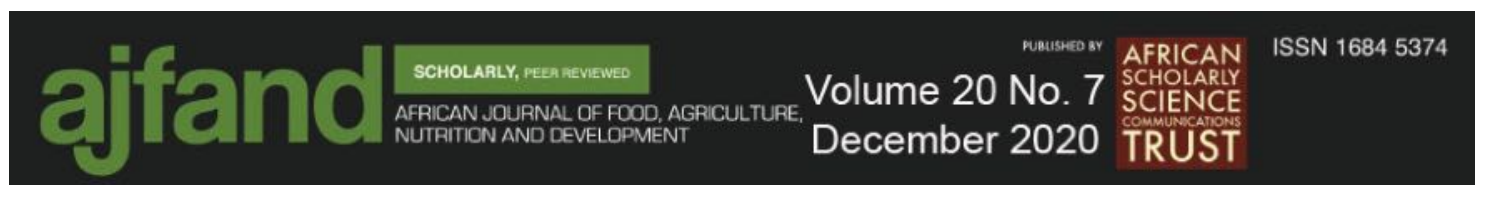

Afr. J. Food Agric. Nutr. Dev. 2020; 20(7): 16941-16956

https://doi.org/10.18697/ajfand.95.18880

A RAPID RESPONSE VACCINE DEVELOPMENT STRATEGY FOR NEWCASTLE DISEASE IN POULTRY

\title{
Maremagae $\mathrm{SR}^{1,2}$, Bezuidenhout $\mathrm{CC}^{2}$, Murphy $\mathrm{AM}^{3}$,
} Jarvis $\mathrm{MA}^{3}$, Naicker $\mathrm{P}^{1}$ and PJ Lebea ${ }^{1,4 *}$

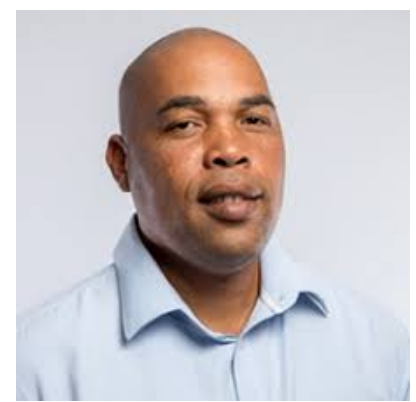

Phiyani J Lebea

*Corresponding author email: phiyani@tokabio.com

${ }^{1}$ Biosciences Unit, Council for Scientific and Industrial Research, Pretoria, South Africa

${ }^{2}$ Unit for Environmental Sciences and Management, Potchefstroom Campus, NorthWest University, Potchefstroom, South Africa

${ }^{3}$ School of Biomedical Sciences, University of Plymouth, Plymouth, Devon, UK

${ }^{4}$ Tokabio (Pty) Ltd, Unit 4, Manhattan Office Park, Centurion, South Africa 


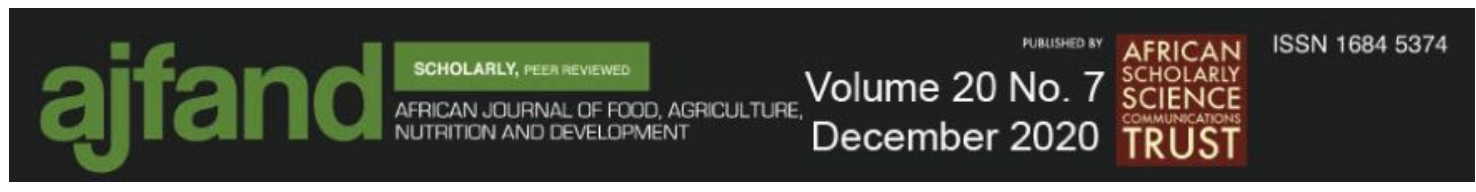

\begin{abstract}
Poultry is a major source of protein in sub-Saharan Africa and many other lower-income regions. Newcastle disease virus (NCDV) comprises a significant threat toward poultry production. While NCDV vaccines are routinely used in developed countries, those used in sub-Saharan Africa are mostly imported and are not specific to locally circulating strains. Indeed, the lack of rapid, field-based NCDV detection and the absence of costeffective production methods for pure, strain-specific vaccines hampers efficient poultry production throughout these regions. This remains a major problem for both subsistence and commercial farming. The aim for this study was firstly, to develop a field-based isothermal PCR assay for NCDV detection that employed a portable instrument and realtime data transfer application. Secondly, to use the nucleic acid sequence data obtained from field isolates to develop a protocol compatible with rapid emergency vaccine production for NCDV. To achieve this, the isothermal PCR detection assay was applied to field isolates from suspected NCDV outbreaks on commercial poultry farms in KwaZulu-Natal, South Africa, while for the vaccine development, the NCDV matrix gene of one of the isolates was sequenced and used to design primers for the recombinant cloning of this antigen into an adenoviral vector. This 'vaccine vector' and a control adenoviral vector were each amplified in 293T cells and then used to infect both 293T cells as a production cell line and chicken embryo fibroblasts (CEF) as a preliminary model of the target host. Western blotting confirmed the successful expression of the V5 epitope tag by the control vector in both cell lines, which established the compatibility of the adenovirus vector as an appropriate carrier of the target antigen. Mass spectrometry confirmed expression of the NCDV matrix protein by the vaccine vector in both cell lines. In conclusion, the improved turnaround time from detection to the production of the vaccine antigen was under 6 weeks. The approach described here provides a rapid and cost-effective protocol for both the pathogen detection on-site and the production of pure vaccine antigens specific to an emerging field strain of NCDV within lower-income regions.
\end{abstract}

Key words: Newcastle disease, poultry, vaccine, adenovirus, isothermal PCR, low and middle income countries (LMICs) 


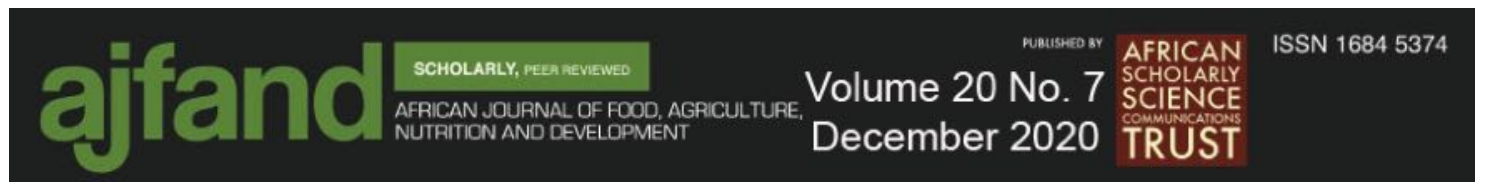

\section{INTRODUCTION}

Poultry production is increasingly recognized as one of the possible solutions to malnutrition, food security and poverty in low- and middle-income countries (LMICs) $[1,2,3]$. Newcastle disease (NCD) is one of the most fatal diseases of birds and a major threat to the poultry industry worldwide [4]. The causative agent is the Newcastle disease virus (NCDV), which is a single-stranded, non-segmented and negative-sense RNA virus that belongs to the Paramyxoviridae family [5]. The NCDVs are classified into three pathotypes based on the severity of the disease they cause. The lentogenic pathotypes are of low virulence [6], the mesogenic pathotypes are moderately pathogenic, thus causing significantly lower mortality [6], while velogenic pathotypes are highly fatal and cause the severest form of NCD [7].

Vaccination remains the primary and most effective strategy for the prevention and management of NCDV [8]. In general, live vaccines are often used for the prevention of NCDV because they exhibit mild symptoms after immunisation [9]. However, live NCDV vaccines frequently exert adverse effects, with respiratory disease and reduced egg production being the most frequently reported symptoms [10]. Live vaccines may also revert to virulence, leading to clinical disease and loss of production.

Owing to the size of the global poultry industry, which includes rural subsistence chicken farming in LMICs, there is consequently a high demand for NCD vaccines. However, velogenic field strains show high genetic diversity, and while cross-protection is often present to some degree, there is no guarantee that commercially available vaccines will confer sufficient (or any) immunity [11]. Most of the NCD vaccines used in sub-Saharan Africa are imported from outside the continent, and are, therefore, not based on strains relevant to the region. In Indonesia, sequence divergence between the field and vaccine strains (Hitchner B1 and LaSota) was shown to be the cause of a severe NCDV outbreak that led to $70 \%$ mortality among the vaccinated birds [12]. The few vaccines that are produced locally are generally impure, containing both viral structural and non-structural proteins, which prevent differentiation between vaccinated and infected animals (DIVA) based on immunological testing.

Human adenoviruses (Ads) are well suited for use as recombinant vaccine vectors. This is because Ads can efficiently infect a wide variety of cell types, have the capacity to grow to high titres in vitro, have high levels of transgene expression, lack integration into the host genome and show genetic stability [13]. Adenovirus vaccines have also been shown to induce potent immune responses in various cell lines [14] as well as in chickens [15], and the efficacy of Ad-based vector vaccines in protecting chickens against virulent strains of avian influenza has been demonstrated [16]. The aim for this study was to develop and use a field detection assay combined with an adenovirus vector-based method for outbreak-specific vaccine production to enable quick turnaround from NCDV detection to disease management. 


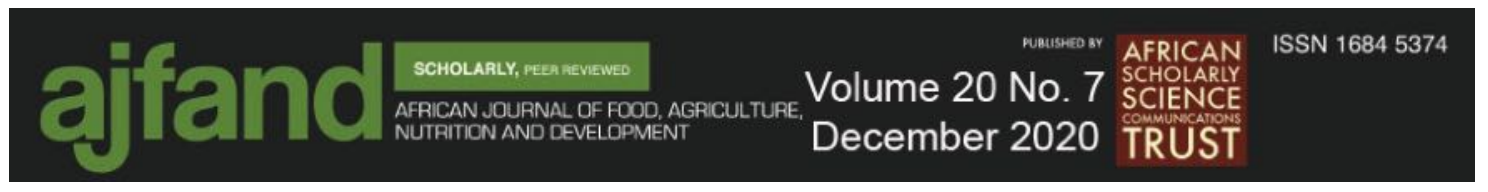

\section{MATERIALS AND METHODS}

\section{Virus detection, vaccine vector design and sequence verification Sample collection and Viral RNA extraction}

Twenty-nine NCDV samples were collected by Rainbow Farms (Pty) Ltd (KwaZuluNatal, South Africa) during suspected outbreaks in their poultry stock in 2016. These samples were isolated within a single month from different farms in KwaZulu-Natal and were likely of the same strain, with a mortality rate of $15-20 \%$. All samples tested positive for NCDV based on PCR analysis performed by Rainbow Farms. The NCDV samples mentioned above were used in the current study. Viral RNA was extracted from a $140 \mu \mathrm{L}$ aliquot of each of the $5 \mathrm{~mL}$ of inactivated NCDV samples with the QIAamp Viral RNA Mini Kit (Qiagen, Valencia, CA, USA) and eluted in $60 \mu \mathrm{L}$ elution buffer according to the manufacturer's instructions. The RNA samples were stored at $-80^{\circ} \mathrm{C}$ for later usage.

\section{Isothermal PCR design and Virus detection}

Isothermal PCR is a rapid nucleic acid amplification assay which takes thirty (30) to sixty (60) minutes at an isothermal temperature of $60-65^{\circ} \mathrm{C}$ (17). For the detection of NCDV, the primers designed by Tian et al. [17] were used as described for isothermal PCR-based detection on an Axxin T16 isothermal PCR instrument (Axxin, Fairfield, VIC, Australia), using a NCDV sample that had previously been characterized as a positive control and buffer only as the negative control. The Axxin instrument was preloaded with an inhouse developed 'ImPulse' software application capable of uploading field results into a designated central database in real-time. Analysis of the data is then carried out by experienced personnel situated anywhere in the world through verified login details provided.

\section{Target gene sequencing and primer design for cloning}

Among the samples tested by isothermal PCR, one sample was randomly chosen for next-generation sequencing. The viral RNA extracted from this sample was reverse transcribed into cDNA with the Transcriptor First Strand cDNA Synthesis Kit (Roche Life Science, Mannheim, Germany) according to the manufacturer's instructions. The whole genome including the NCDV matrix gene was sequenced in-house using the Ion Torrent TM Personal Genome Machine (PGM ${ }^{\mathrm{TM}}$ ) next generation sequencer (Thermo Fisher Scientific, Johannesburg, South Africa).

PCR was chosen to amplify the longest possible matrix gene fragment for cloning purposes. The NCBI Primer-BLAST tool was used to design six primer sets (Table S1Supplementary data) for amplification of the NCDV matrix gene from the isolated viral RNA, based on apparent conserved sites across the sequenced matrix gene (Fig. S1Supplementary data) and the matrix genes of NCDV strains deposited in the NCBI database. Based on this analysis, the forward primer MF8A (5'ATAGGATCCAGCGCCTTGAC-3') and the reverse primer MR5 (5'GTATTTGGCAAGGGTGTGCC-3') were selected, as this primer set amplified the longest fragment (956bp) between conserved sites compared to the other possible primer sets (Fig. S2- Supplementary data). 


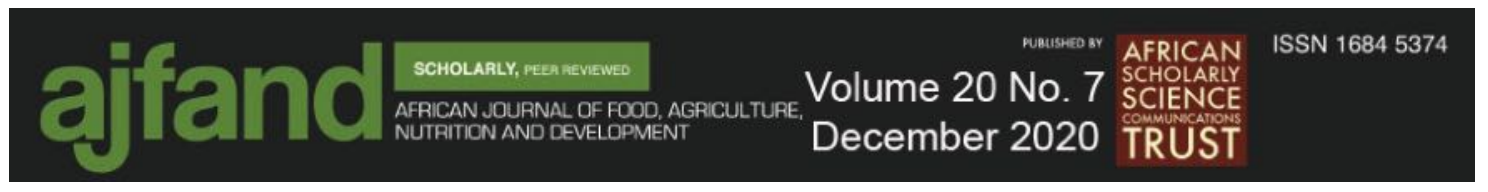

\section{Recombinant cloning}

To amplify the NCDV matrix gene fragment for subsequent cloning, the KAPA HiFi HotStart ReadyMix PCR Kit (KAPA Biosystems, Cape Town, South Africa) was used according to the manufacturer's recommendations. The PCR reaction comprised $12.5 \mu \mathrm{L}$ of $2 \times$ KAPA HiFi HotStart ReadyMix, $0.6 \mu \mathrm{M}$ each of the MF8A and MR5 primers and $11 \mu \mathrm{L}$ of cDNA prepared from the viral RNA (total volume per reaction, $25 \mu \mathrm{L}$ ). The PCR was performed with the Roche LightCycler 480 instrument using the recommended cycling parameters with the annealing temperature maintained at $57^{\circ} \mathrm{C}$. The PCR products were resolved by $1 \%$ agarose gel electrophoresis to confirm amplification and were extracted from the agarose gel with the GeneJET Gel Extraction Kit (Thermo Fisher Scientific).

The amplified NCDV matrix gene fragment was cloned into an adenoviral expression vector (pAd/CMV/V5-DEST) using the pAd/CMV/V5-DEST Gateway Vector Kit (Thermo Fisher Scientific) according to the manufacturer's instructions. Briefly, a blunt-end PCR product (to ensure directional cloning) was generated from the amplified matrix gene fragment with appropriately designed primers (MBf: 5'CACCATGTATAGGATCCAGCGCCTTGAC-3', MBr: 5'-

GTATTTGGCAAGGGTGTGCC-3'). The gel-cleaned blunt-end PCR product and the pENTR/D TOPO vector (Thermo Fisher Scientific) were mixed together at room temperature for $5 \mathrm{~min}$. This mixture was transformed into competent $E$. coli cells. Plasmid DNA was isolated from the positive transformants and used in the LR recombination reaction with the $\mathrm{pAd} / \mathrm{CMV} / \mathrm{V} 5-\mathrm{DEST}$ vector. The LR recombination reaction mixture was then transformed into competent $E$. coli cells, followed by purification of the adenoviral vector from the positive transformants.

\section{Recombinant adenovirus propagation}

The purified recombinant adenoviral plasmid DNA was first digested with the PacI enzyme (Thermo Fisher Scientific) according to the manufacturer's instructions before transfection into the human embryonic kidney (293T) cells (Thermo Fisher Scientific). Approximately $5 \times 10^{5} 293 \mathrm{~T}$ cells were seeded per well in a 6-well plate containing 2 $\mathrm{mL} /$ well of Dulbecco's Modified Eagle Medium (DMEM; Lonza, Kempton Park, South Africa) supplemented with $10 \%$ foetal bovine serum (FBS; Thermo Fisher Scientific) and $0.05 \mathrm{mg} / \mathrm{L}$ gentamicin (Lonza). After a 24-h incubation, the cells were transfected with the digested adenoviral vector using Lipofectamine 2000 (Thermo Fisher Scientific). Once a cytopathic effect of $80 \%$ was observed, the adenovirus was harvested from the cells using the freeze and thaw method. The resultant viruses were then stored at $-80^{\circ} \mathrm{C}$. The Ion PGM system was used to confirm the sequence of the target NCVD matrix gene insert in the recombinant adenoviral vector.

Expression of the control and recombinant adenovirus vectors in $293 \mathrm{~T}$ cells and chicken embryo fibroblasts (CEFs)

Preparation and infection CEFs

Chicken Embryo Fibroblasts (CEFs) were prepared from 11-day-old chicken eggs according to a standard protocol [18]. These cells were then incubated at $37^{\circ} \mathrm{C}$ with $5 \%$ $\mathrm{CO}_{2}$ at a set humidity of $95 \%$. At the third passage, the cells were visualised with an Olympus light microscope BX53 (Olympus Corporation, Tokyo, Japan) using phase 


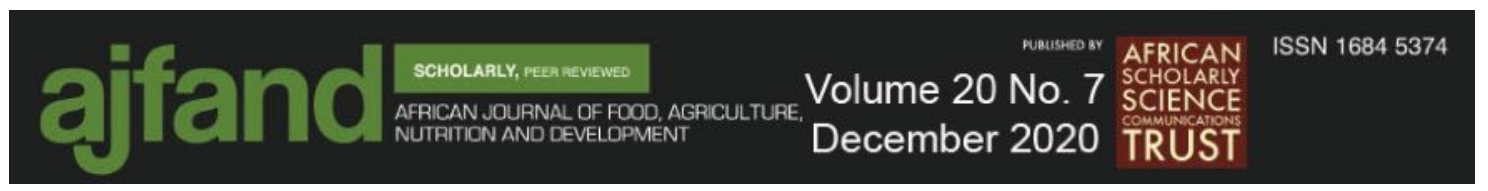

contrast at $10 \times$ magnification. As a preliminary investigation of the infectivity of adenovirus towards the targeted host (chicken), a control adenovirus vector (pAd/CMV/V5-GW/lacZ; Thermo Fisher Scientific) and the recombinant adenovirus vector (the "vaccine vector") were used to infect the CEFs. Approximately $3 \times 10^{6} \mathrm{CEFs}$ were suspended in $10 \mathrm{~mL}$ DMEM supplemented with $10 \%$ FBS and seeded per $10-\mathrm{cm}$ tissue culture plate followed by incubation as before. At 70\% confluency, cells in one set of plates were infected with the purified control adenovirus (pAd/CMV/V5-GW/lacZ) containing theV5 epitope, while the cells in the other set of plates were left uninfected (negative control). Cells were incubated for 4 days, following which, the cells were visualised by light microscopy to check for any signs of cytopathic effect following viral infection.

The $293 \mathrm{~T}$ cells were also infected with the control vector by following the same infection protocol used for the CEFs.

\section{Western blot analysis of the expression of the control adenovirus vector in 293T cells and CEFs}

To test the compatibility of the adenovirus vector with the CEF primary cell line, the expression of the V5 epitope in infected cells was determined through western blot analysis. Briefly, after cell incubation for 4 days following infection, total cell lysates were prepared using lysis buffer ( $1 \mathrm{M}$ Tris $\mathrm{pH} 8.0,0.5 \mathrm{M}$ EDTA, $1 \mathrm{M} \mathrm{KCl}, 0.5 \%$ Triton $\mathrm{X}-100$ and $1 \times$ protease inhibitor) and subjected to SDS-PAGE on a Bolt $4-12 \%$ Bis-Tris Plus gel (Thermo Fisher Scientific) and then the proteins were transferred to an ImmuneBlot PVDF Membrane (Bio-Rad, Johannesburg, South Africa). The membrane was blocked in PBS-T $(1 \times$ PBS pH 7.4, 0.1\% (v/v) Tween 20) containing 5\% non-fat dry milk (Bio-Rad) for $2 \mathrm{~h}$ at $4^{\circ} \mathrm{C}$. The membrane was rinsed three times with PBS-T and incubated overnight at $4^{\circ} \mathrm{C}$ with mouse anti-V5 (AbD Serotec, Hercules, CA, USA) diluted 1:2,000 in 5\% milk PBST. After three rinses with PBS-T, the membrane was incubated with 1:2,000 goat anti-mouse IgG-HRP (Santa Cruz Biotechnology, Heidelberg, Germany) in 5\% milk PBS-T for a $1 \mathrm{~h}$ at room temperature. The chemiluminescent signal was detected by applying the Clarity ECL substrate (Bio-Rad) and exposing the membrane to UV light. Images were captured with the Gel Doc XR+ documentation system (Bio-Rad). The same procedure was followed to analyse the expression of the V5 epitope in the 293T cells.

\section{Mass spectrometry analysis of the expression of the recombinant adenovirus vector (Ad-recNCDV) in CEFs}

Following confirmation of the expression of the control adenovirus vector in 293T cells and CEFs using western blots, expression of the NCDV matrix protein from the AdrecNCDV was analysed by mass spectrometry for the purpose of establishing a rapid emergency vaccine development protocol. Using standard procedures, protein was extracted from the non-infected CEFs and 293T cells (negative controls) and the CEFs infected with the vaccine vector. Protein samples $(20 \mu \mathrm{g}$ each) were purified of salts and detergents with MagReSyn HILIC microparticles (ReSyn Biosciences, Pretoria, South Africa) with on-particle protein digestion using sequencing-grade modified trypsin (Promega, Madison, WI, USA). The resultant peptides were analyzed with a Dionex UltiMate 3000 RSLC system (Thermo Fisher Scientific) coupled to an AB Sciex 


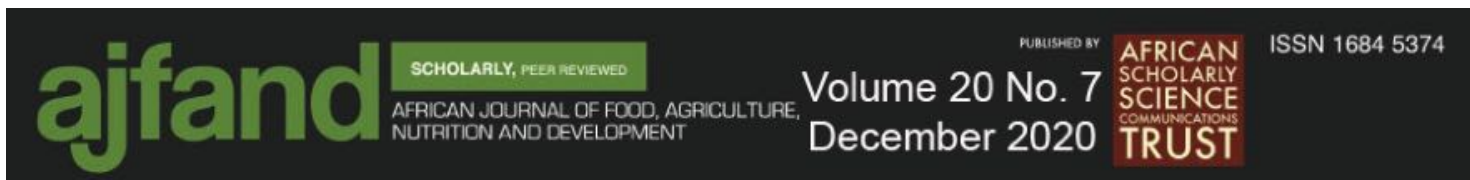

TripleTOF 6600 mass spectrometer (AB Sciex, Singapore). Mass spectrometry (MS) data were searched with the SearchGUI interface [19] using the X!Tandem [20], MS$\mathrm{GF}+[21]$ and Comet [22] search engines. The target database was generated by adding the sequences of the NCDV matrix protein and common contaminants to the whole proteomes of dsDNA viruses listed in the UniProt database. Decoy sequences were created by reversing all the sequences in the target database in SearchGUI. A 1\% false discovery rate cut-off was applied at the PSM (peptide-spectrum match) and protein level.

\section{RESULTS AND DISCUSSION}

Field-based virus detection, vaccine vector design and sequence verification

The isothermal PCR successfully detected NCDV in the 29 field cases, previously confirmed positive using a conventional PCR assay. The results on the axxin instrument are shown in a graph format where a sigmoidal curve shows that the sample tested is NCDV positive, while a straight line shows negative results. Only three of the samples tested with the isothermal PCR are shown in Fig. 1 and these samples were confirmed to be NCDV positive. The total time for sample preparation and virus detection, which included the 30-minute sample run on the Axxin T16 instrument, was less than 90 min. The samples were detected by isothermal PCR within 15 minutes of completing virus extraction.

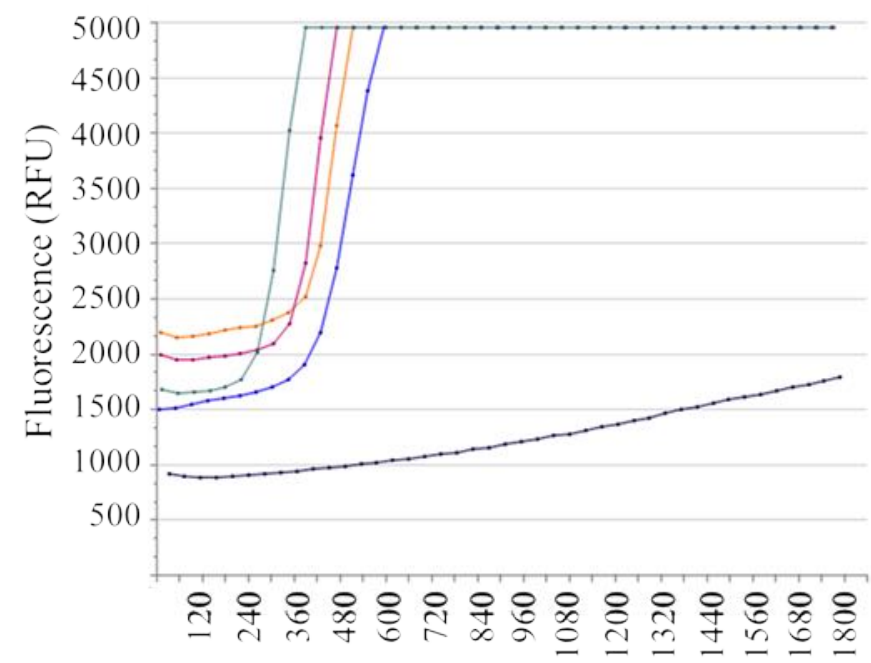

Time $(s)$

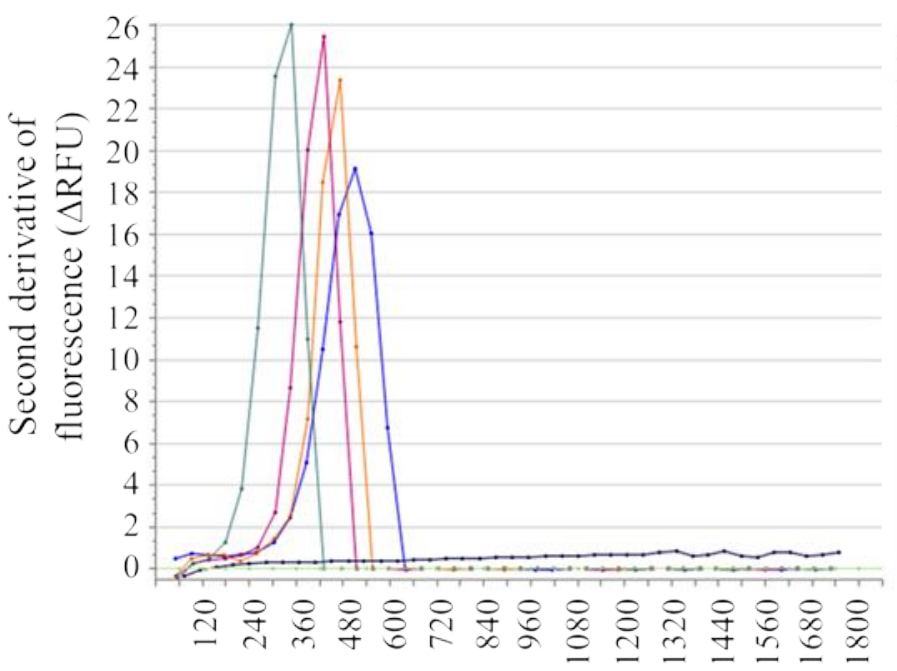

Time $(s)$

Figure 1: Newcastle disease samples were detected by isothermal PCR targeted towards the matrix gene. (A) Fluorescence signal over time during isothermal PCR of the positive control (green), the field samples (red, yellow and blue) and the negative control (black). (B) The second derivative plot of the data in (A) to pinpoint the exact time of identification (peaks) within the 30 -minute assay run

Antibodies against the NCDV hemagglutinin-neuraminidase $(\mathrm{HN})$, fusion glycoproteins and matrix gene products are produced following infection with NCDV [23]. The 


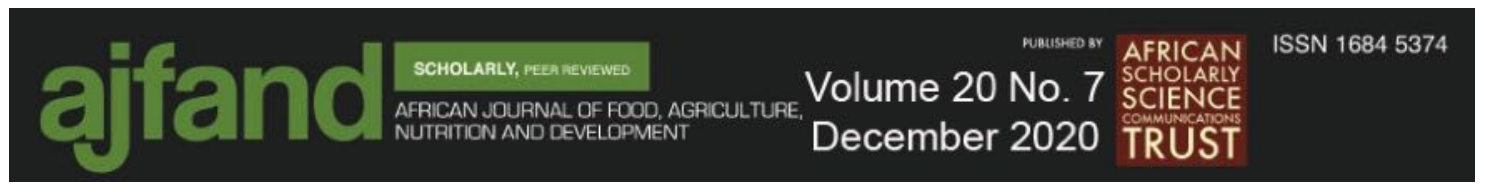

antibodies formed against each of these proteins, particularly $\mathrm{HN}$, have been used successfully to protect chickens against virulent NCDV strains [24]. For the present study, the matrix gene was, however, chosen as the detection marker and vaccine antigen since it is the most widely used detection target for both immunological and molecular tests (such as ELISA and PCR). To validate our isothermal PCR method, it had to be comparable with the methods commonly used for the detection of NCDV. The same method developed here for the matrix gene could later be adapted toward the more immunologically protective $\mathrm{HN}$ and fusion glycoprotein genes, either singly or in combination for maximum potency as a vaccine. However, using the latter two genes as a model from the beginning would have resulted in incongruent validation of the detection method.

\section{Expression of the adenovirus control and vaccine vectors in CEFs}

Before a vector can be used as a carrier of the gene of interest, it is important to verify whether it can infect the chosen production cell line as well as the targeted host primary cell line. In pursuit of this objective, CEFs were prepared from chicken embryos (Fig. 2) as a preliminary model of the eventual vaccine recipient (chickens). Microscopy confirmed that the fibroblasts were viable and had the expected morphology, without any obvious signs of contamination.

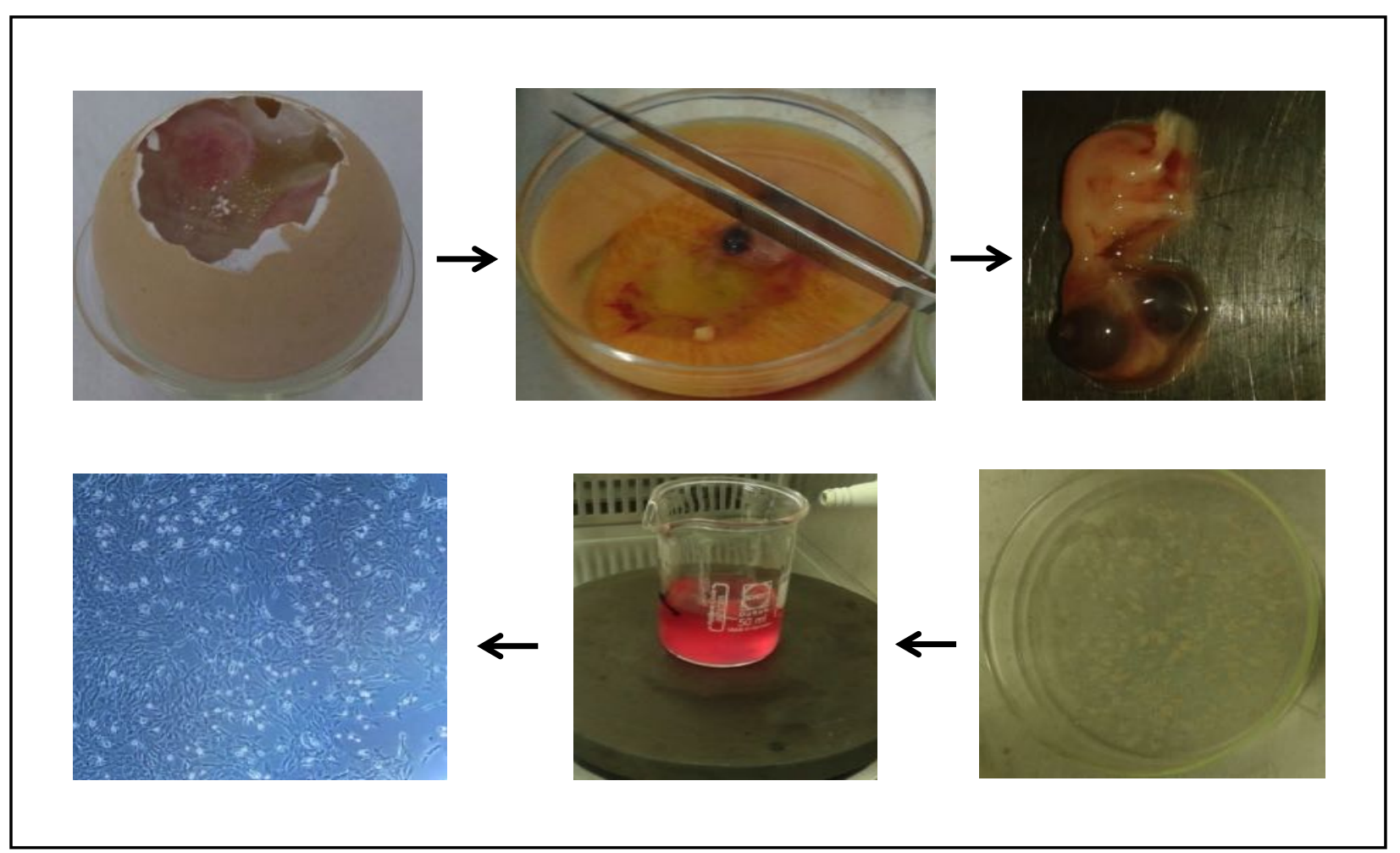

Figure 2: Chicken embryo fibroblasts prepared from an 11-day-old chicken embryo and used for transfection with the recombinant adenovirus vaccine vector. The resulting fibroblasts remained viable after preparation as observed at $90 \%$ confluency $(10 \times$ magnification $)$ 


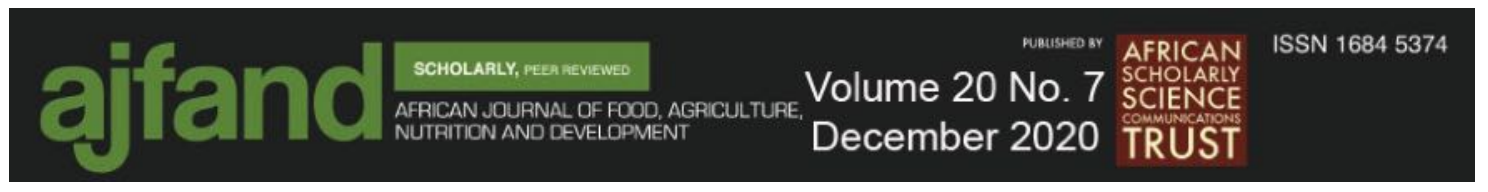

The expression of adenovirus genes in 293T cells has already been reported [25]; therefore, this was performed as a confirmatory step. However, expression of the adenovirus genes in the CEFs was analysed to confirm the suitability of using an adenovirus vector in chickens as the eventual vaccine recipient. The expression of the V5 epitope as a control experiment in both 293T cells and CEF confirmed that the adenovirus is a suitable delivery vector for chickens (Figure 3). Given that 293T cells contain the E1 replication factor, expression of the V5 epitope was higher, confirming that $293 \mathrm{~T}$ cells can be used as a production cell line. Expression of the V5 epitope was much weaker in CEFs as these cells lack the E1 gene thus rendering adenovirus replication deficient in these cells [25].

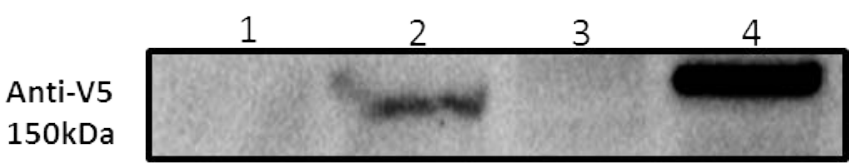

Figure 3: Western blot analysis of the control adenovirus vector V5 epitope in 293 T cells and CEFs. Lane 1, uninfected CEFs; lane 2, CEFs infected with the adenovirus vector; lane 3 , uninfected $293 \mathrm{~T}$ cells; and lane 4, $293 \mathrm{~T}$ cells infected with the adenovirus vector

Expression of the NCDV matrix gene by the vaccine vector was analysed in the CEFs. Since it is critical that expression of the gene of interest be directly confirmed so as to inform decision-making during the vaccine development process, mass spectrometry (MS) was used. Figure 4 shows regions in the matrix protein that were digested by the trypsin enzyme to form the peptides analysed by MS. Five peptides were identified from viruses propagated in the $293 \mathrm{~T}$ cells with a NCDV matrix protein coverage of $19 \%$ (Table 1). Most importantly, MS also confirmed the expression of the NCDV matrix gene by the recombinant adenovirus in the CEFs, where four peptides were identified with a NCDV matrix protein (Table 1). Generally, identification of peptide sequences in one region of a single protein after trypsin digestion of the protein of interest is enough to positively identify such a protein. In this case, four different pieces of peptide sequences were identified. These 4 pieces of peptide sequences were equal to $16 \%$ of the total protein length, hence the $16 \%$ coverage. This conclusively identifies the matrix protein as being present in the developed vaccine vector. Therefore, these percentage coverages are significant enough to consider the matrix protein to be expressed in both cell lines. As anticipated, non-infected CEFs contained none of the peptides identified in the matrix protein. A single unique peptide is usually enough to identify a specific protein presence. In this case, no less than four (4) fragments were identified in 293T cells and CEFs that were uniquely those of the matrix gene protein product. These results indicate that the recombinant adenovirus vector could function as a vaccine production system and that $293 \mathrm{~T}$ cells could be used as a host cell line for virus propagation. 


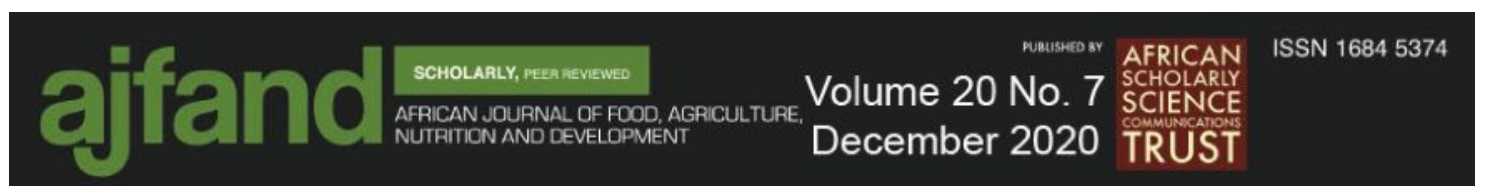

YRIQRLDLWTDSKEDSVFITTYGFI FQVGNEEATVGI I DDKPKRELLSAAMLCLGSVPNT GDLIELARACLTMMVTCKKSATNTERMVFSVVQAPQVLQSCRVVANKYSSVNAVKHVKA EKIPGSGTLEYKVNFVSLTVVPKKLDYKIPAAVLKISGSSLYNLALNVTINVEVDPRSPL VKSLSKSDSGYYANLFLHIGLMTTVDRKGKKVTFDKLEKKIRSLDLSVGLSDVLGPSVLV KARGARTKLLAP FFSSS GTACYP IANAS PQVAKILWSQTACLRSVKII I QA GTQRAVAVT ADHEVTSTKLEKGHTLAKY

Figure 4: The NCDV matrix protein sequence cloned into the recombinant adenovirus vector. The underlined amino acid sequence shows where the protein was cleaved during on-particle protein digestion using trypsin. The highlighted regions indicate the peptides identified by mass spectrometry

\section{Suitability of the emergency vaccine production protocol}

Outbreaks caused by NCDV frequently lead to significant economic losses to the poultry industry worldwide [4]. However, current commercial vaccines are not always designed based on strains relevant to all regions $[9,26]$. The current work, therefore, focused on developing a strategy for the rapid production of emergency vaccines specific to outbreak strains emerging in LMICs. Critical to such a strategy is the speed at which the outbreak strain is identified, and a response vaccine is produced. As shown in Figure 5, the entire process described here from initial field identification of the 'outbreak' strain through to the production of the Ad-based vaccine would take a maximum of 6 weeks, which is a significant improvement over conventional strategies that can take 6-8 months [27].

The proposed strategy is cost-effective [28] and can be readily implemented in the subSaharan African region. These emergency vaccines would be designed based on the actual isolated field strains and are, therefore, likely to provide more effective immune protection; while vaccination remains the most successful strategy for the prevention and management of NCD, commercial vaccines are typically at most only mildly effective against local and emerging strains of NCDV. 

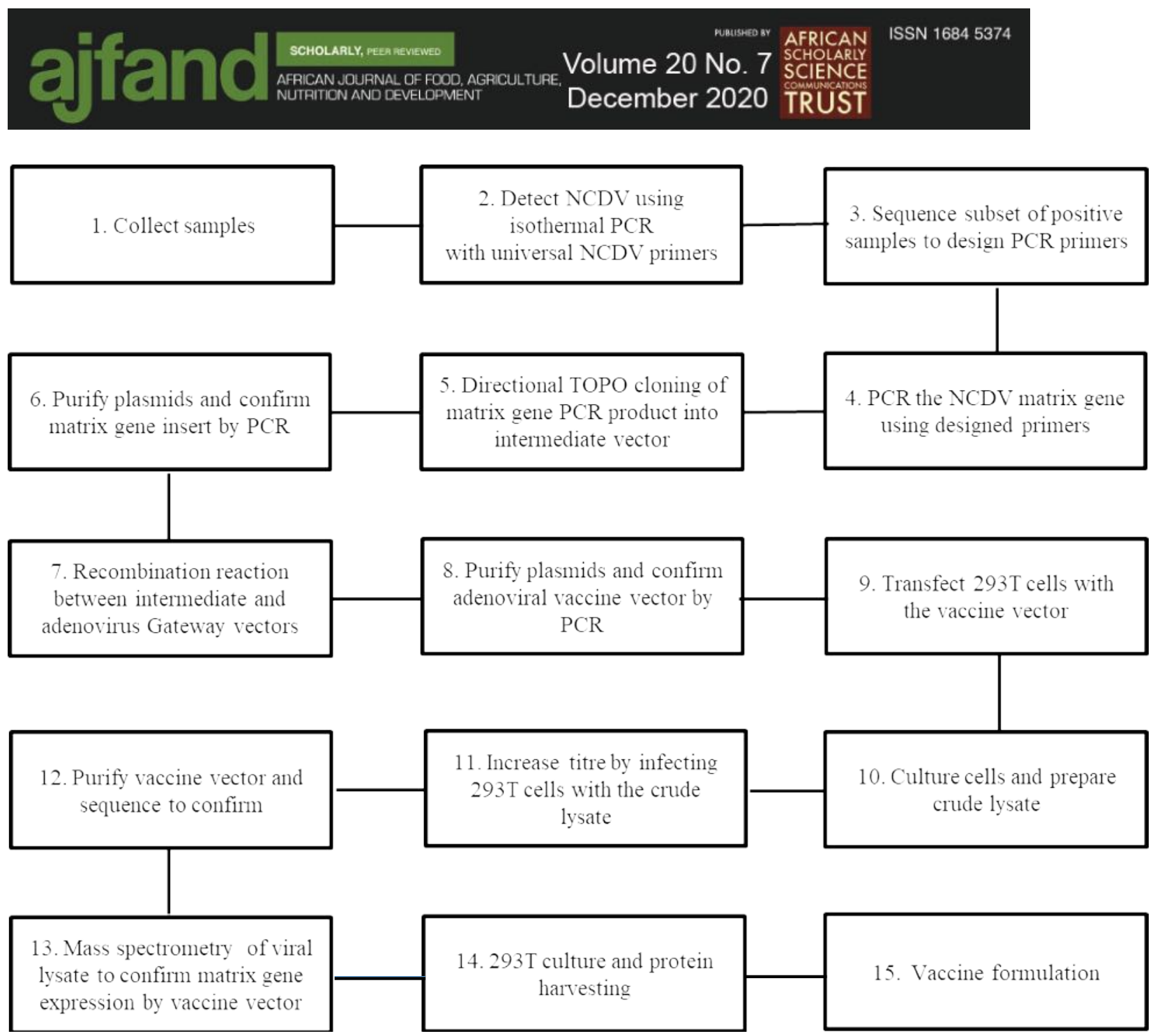

Figure 5: Schematic diagram of the proposed strategy for directed NCD emergency vaccine production, describing the workflow and validation steps from sample isolation to vaccine formulation. The entire process takes less than 6 weeks

Considering that an emergency vaccine would not have the opportunity to undergo clinical trials before being applied in an emergency situation, the emphasis in the current work was equally placed on the use of a specific target antigen and the rapid turnaround of emergency vaccine production. The emergency vaccine was designed using the human recombinant adenovirus vector, since such vectors are known for their ability to infect a wide variety of cell types, to grow to high titres in vitro, to have high levels of transgene expression and their inability to integrate into the host genome [13]. To aid the universal application of our strategy, mass spectrometry was used instead of western blotting to confirm the expression of the target antigen by the recombinant adenovirus, because mass spectrometry is not dependent on the availability of commercial antibodies towards the antigen of interest. While mass spectrometry may not necessarily be cheap or readily available, such analysis can be performed by designated laboratories at a relatively reasonable cost. The combination of the accuracy and fast turnaround of mass spectrometry dictates its preferential inclusion in this strategy. 


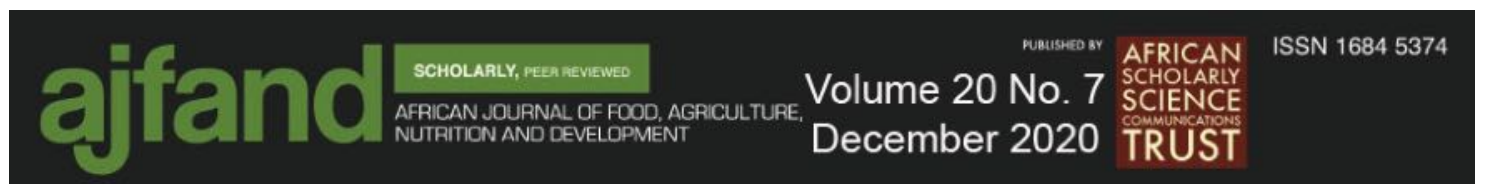

In this model, once an emerging strain is identified, an Ad-based platform allows for the differentiation between vaccinated and infected animals (DIVA) [29]. Ad-based vaccine development allows for ease of genetic manipulation and safety for production in cell lines [25, 30]. Furthermore, adenovirus vector-based vaccines with suitable gene products have also been shown to protect chickens against virulent strains of avian influenza [16]. Although the current study focused on developing an emergency vaccine design and production model and did not investigate the level of immune protection provided by the resultant product, in vivo studies/clinical trials of the recombinant adenovirus vaccine could be performed in live chickens to confirm protection from NCDV infection. However, given that the matrix gene is not necessarily a highly effective immunogenic factor in NCDV protection, more functional genes such as the $\mathrm{HN}$ and $\mathrm{F}$ genes could be inserted in future. The final formulation of the vaccine could also include adjuvants such as short and long-term initiation factors to further improve vaccine efficacy.

\section{CONCLUSION}

This study addresses the current mismatch between existing commercial vaccines and the vaccines required to target emerging NCDV strains, particularly in LMICs. Importantly, this vaccine design approach will likely also prove suitable for other poultry viral diseases besides NCDV. Overall, this new strategy could enable quicker turnaround times for disease management, and it could equip developing nations with the capacity to produce vaccines specific to pathogenic strains of NCDV emerging within their region. In this way it will contribute towards food security in the regions that require it the most.

\section{ACKNOWLEDGEMENTS}

The authors would like to thank Rainbow Farms (Ms Pra Ori) for their kind gift of inactivated Newcastle disease virus strains. Natasha Beeton-Kempen, $\mathrm{PhD}$, edited several drafts of this manuscript. This work was supported by funding from the National Department of Science and Technology of South Africa (DST-RSA) and the Technology Innovation Agency (TIA).

\section{Author contributions}

S.R.M and P.J.L designed the study. S.R.M conducted the experiments. S.R.M and P.J.L analysed, interpreted the data and wrote the manuscript. C.C.B read and approved the manuscript. M.J and AMM advised on the adenovirus work, read and approved the manuscript. P.N assisted with designing and conducting the MS experiments and analysed the MS data.

\section{Ethics approval}

All the experimental protocols involving the chicken embryo fibroblasts were approved by the Council for Scientific and Industrial Research (CSIR) Research Ethics Committee (approval number: 73/2013).

\section{Competing interests}

The authors declare that they have no competing interests. 


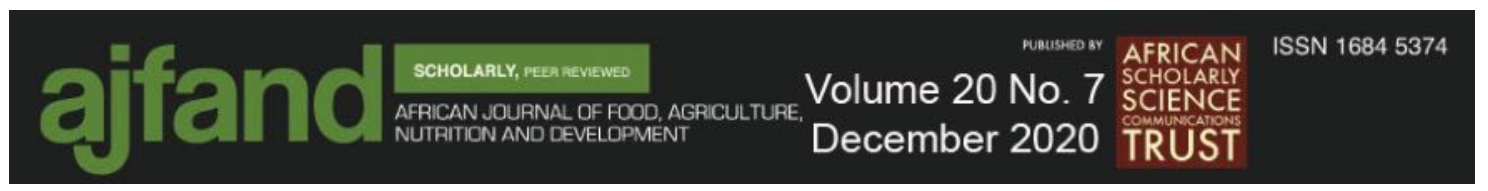

Table 1: Mass spectrometry identification of the NCDV matrix protein expressed by the purified recombinant adenovirus (the "vaccine vector") in $293 \mathrm{~T}$ cells and chicken embryo fibroblasts (CEFs)

\begin{tabular}{lccc}
\hline & $\begin{array}{c}\text { Recombinant } \\
\text { Adenovirus }\end{array}$ & $\begin{array}{l}\text { Infected } \\
\text { CEFs }\end{array}$ & $\begin{array}{c}\text { Non-infected } \\
\text { CEFs (control) }\end{array}$ \\
\hline Matrix protein coverage (\%) & 19 & 16 & - \\
No. of unique peptides identified & 5 & 4 & - \\
\hline Peptides identified (+) & + & + & - \\
\hline N-APEKIPGSGTLEYK-C & + & + & - \\
N-VNFVSLTVVPK-C & + & - & - \\
N-ILWSQTACLR-C & + & + & - \\
N-IIIQAGTQR-C & + & + & \\
N-AVAVTADHEVTSTKLEK-C & + & & - \\
\hline
\end{tabular}




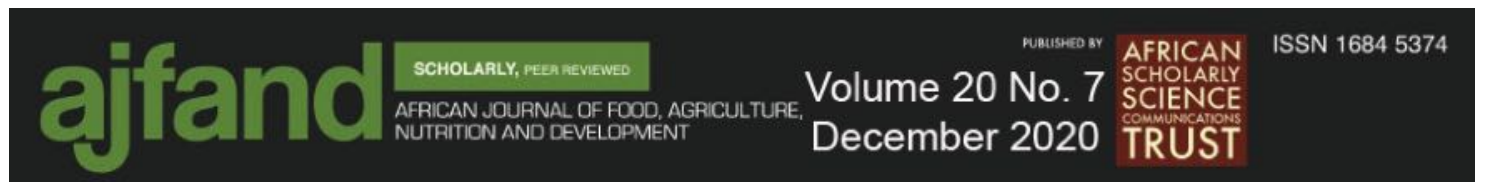

\section{REFERENCES}

1. Pitt M, Shahidur RK, Omar HC and D Millimet Credit programmes for the poor and the health status of children in rural Bangladesh. IER 2003; 44(1): 87118.

2. Fasina FO, Wai MD, Mohammed SN and ON Onyekonwu Contribution of poultry production to household income: a case of Jos South local government in Nigeria. Fam. Poult. 2007; 17(1\&2):30-34.

3. Dei HK, Alidu I, Otchere EO, Donkoh A, Boa-Amponsem K and I Adam Improving the brooding management of local guinea fowl (Numida meleagris). Fam. Poult. 2009; 18(1\&2): 3-8.

4. Miller PJ, Decanini EL and CL Afonso Newcastle disease: evolution of genotypes and the related diagnostic challenges. I Infect Genet Evol. 2010; 10: 2635 .

5. Amarasinghe GK, Báo Y, Basler CF, Bavari S, Beer $M$ and $\mathbf{N}$ Bejerman Taxonomy of the order Mononegavirales: update:2017 Arch virol. 2017; 162: 2493-504.

6. Moura VMBD, Susta $\mathbf{L}$ and S Cardenas-Garcia Neuropathogenic capacity of lentogenic, mesogenic and velogenic Newcastle disease virus strains in day-old chickens. Vet pathol.2016; 53(1): 53-64.

7. Piacenti AM, King DJ, Seal BS, Zhang J and CC Brown Pathogenesis of Newcastle disease in commercial and specific pathogen-free turkeys experimentally infected with isolates of different virulence. Vet pathol.2006; 43(2): 168-178.

8. Barman LR, Islam MN, Flensburg MF, Permin A, Petersen SL and MR Islam Newcastle disease vaccination regimen comprising both lentogenic and mesogenic strains is more effective than lentogenic strain only. The Bangladesh Veterinarian. 2010; 27 (1): 1-7.

9. Alexander DJ, Bell JG and RG Alders Technology review: Newcastle disease with special emphasis on its effect on village chickens. Food and agriculture organization of the united nations Rome. FAO Animal Production and Health series 2004: 161.

10. Dimitrov KM, Afonso CL, Yu Q and PJ Miller Newcastle disease vaccines - A solved problem or a continuous challenge? Vet. Microbiol. 2017; 206:126-136.

11. Miller PJ, Afonso CL, Attrache JE, Dorsey KM, Courtney SC, Guo Z and DR Kapczynski Effects of Newcastle disease virus vaccine antibodies on the shedding and transmission of challenge viruses. Dev Comp Immunol 2013; 41: 505-513. 


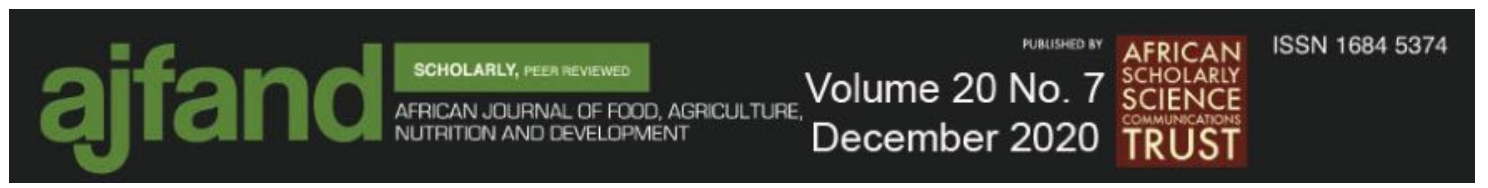

12. Xiao S, Nayak B, Samuel A, Paldurai A, Kanabagattebasavarajappa M, Prajitno TY, Bharoto EE, Collins PL and SK Samal Generation by reverse genetics of an effective, stable, live-attenuated Newcastle disease virus vaccine based on a currently circulating, highly virulent Indonesian strain. PLos ONE.2012; 7 (12).

13. Robert-Guroff $\mathbf{M}$ Replicating and non-replicating viral vectors for vaccine development. Curr. Opin. Biotech. 2007; 18:546-556.

14. Banchereau J and RM Steinman Dendritic cells and the control of immunity. Nature. 1998; 392: 245-252.

15. Singh S, Toro H, Tan g D, Briles WE, Yates LM, Kopulos RT and EW Collison Non-replicating adenovirus vectors expressing avian influenza virus hemagglutinin and nucleocapsid proteins induce chicken specific effector, memory and effector memory CD8+ T lymphocytes. Virology.2010; 405: 62-69.

16. Gao W, Soloff AC, Lu X, Montecalvo A, Nguyen DC, Matsuoka Y, Robbins PD, Swayne DE, Donis RO, Katz JM, Barratt-Boyes SM and A Gambotto Protection of mice and Poultry from lethal H5N1 avian influenza virus through adenovirus-based immunization. J.Virol. 2006; 80(4): 1959-1964.

17. Tian B, Ma J, Zardán Gómez de la Torre T, Bálint Á, Donolato M, Hansen MF and M Strömberg Rapid Newcastle disease virus detection based on Loopmediated isothermal amplification and optomagnetic readout. Sensors 2016; 1(10): $1228-1234$.

18. Hernandez $\mathbf{R}$ and DT Brown Growth and maintenance of chick embryo fibroblasts. Curr. Protoc. Microbiol. 2010; 17: A.41.1-A.41.8.

19. Barsnes $\mathbf{H}$ and $\mathbf{M}$ Vaudel SearchGUI: A highly adaptable common interface for proteomics search and de novo engine. J. Proteome Res. 2018; 17(7): 2552-2555.

20. Craig R and RC Beavis TANDEM: matching proteins with tandem mass spectra. Bioinformatics 2004; 20(9): 1466-1467.

21. Kim S and PA Pevzner MS-GF+ makes progress towards a universal database search tool for proteomics. Nat. Commun. 2014; 5: 5277.

22. Eng JK, Jahan TA and MR Hoopman Comet: an open-source MS/MS sequence database search tool. Proteomics 2013; 13(1): 22-24.

23. Panshin A, Shihmanter E, Weisman Y, Orvell $\mathbf{C}$ and $M$ Lipkind Antigenic epitope characterisation of matrix protein of Newcastle disease virus using monoclonal antibody approach: contrasting variability amongst NDV strains. Comp Immunol. Microbiol. Iinfect. Dis. 1997; 20(2):177-189. 


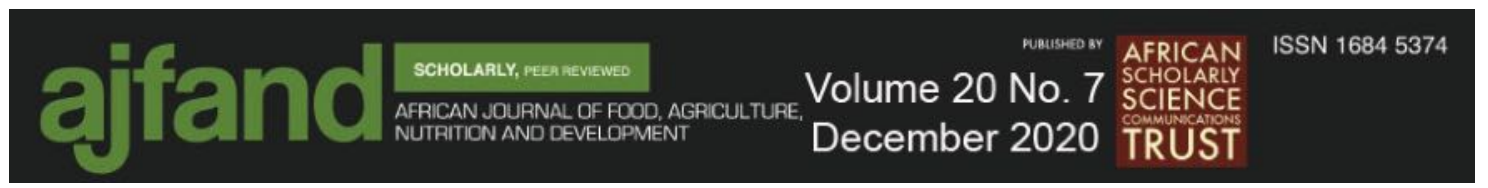

24. Kamiya N, Niikura M, Ono M, Kai C, Matsuura Y and T Mikami Protective effect of individual glycoproteins of Newcastle disease virus expressed in insect cells: the fusion protein derived from an avirulent strain had lower protective efficacy. Virus Res. 1994; 32: 373-379.

25. Graham FL, Smiley J, Russel WC and R Nairn Characteristics of a human cell line transformed by DNA from human adenovirus type 5. J. Gen. Virol. 1977; 36: $59-72$.

26. Bello MB, Yusoff KM, Ideris A, Hair-Bejo M, Peeters BPH, Jibril AH, Tambuwal FM and AR Omar Genotype diversity of Newcastle disease virus in Nigeria: Disease control challenges and future outlook. Adv virol. 2018; Article ID D6097291.

27. Choi Y and J Chang Viral vectors for vaccine applications. CEVR 2013; 2: 97 105.

28. Milián E and AA Kamen Current and emerging cell culture manufacturing technologies for influenza vaccines. Biomed Res, Int 2015; 11 pages. Article ID: 504831): https://doi.org/10.1155/2015/504831

29. Capua I, Terregino C, Cattoli G, Mutinelli F and JF Rodriguez Development of a DIVA (differentiating infected from vaccinated animals) strategy using a vaccine containing a heterologous neuraminidase for the control of avian influenza. Avian Pathol. 2003; 32: 47-55

30. Fallaux FJ, Bout A, Van der Velde I, Van den Wollenberg DJ, Hehir KM and J Keegan New helper cells and matched early region 1-deleted adenovirus vectors prevent generation of replication-competent adenoviruses. Hum. Gene Ther. 1998; 9: 1909-1917. 